\title{
el quinto symposio internacional de química del cemento
}

Dr. J. CALLEJA

\section{GENERALIDADES}

Entre los días 6 y 12 de octubre de 1968, y patrocinado por la Asociación de Fabricantes de Cemento del Japón, ha tenido lugar en Tokyo el Quinto Symposio Internacional de Química del cemento (V-ISCC).

Se ha desarrollado bajo los auspicios de la Agencia de Ciencia y Tecnología, y del Ministerio de Industria y Comercio Internacional, del Japón. Ha sido sufragado por los siguientes organismos japoneses: Consejo de la Ciencia, Asociación de Cerámica, Sociedad de Ingenieros Civiles, Instituto de Arquitectos y Sociedad de Química.

La Organización ha corrido a cargo de un Comité formado por un Presidente y dos Vicepresidentes pertenecientes todos a la Industria del Cemento, y dos Vicepresidentes más, uno perteneciente a la Universidad de Tokyo y otro a la Agencia de Ciencia y Tecnología; dieciocho miembros integrantes del grupo de asistencia a la Dirección, todos los cuales pertenecían a empresas productoras de cemento y algunas de productos químicos; tres miembros integrantes del grupo de Personal del Symposio, pertenecientes a la industria del cemento; dos miembros del grupo adscrito al Secretariado, pertenecientes a la Asociación de Fabricantes de Cemento; y quince consejeros más, pertenecientes a Universidades e Institutos Tecnológicos, e industrias del cemento y química. Ostentó el Secretariado un miembro de la Asociación de Fabricantes de Cemento.

Los actos del Symposio se desarrollaron en el Palace Hotel y en el Metropolitan Festival Hall de Tokyo. Además de los actos de apertura (día 6) y de clausura (día 11), y de las sesiones de trabajo, tuvieron lugar, al final (día 12), visitas técnicas a fábricas de cemento y laboratorios de investigación. En la tarde del día 9 se intercaló una gira industrial de Tokyo (fábricas SONY de aparatos electrónicos y CANON de cámaras fotográficas).

Las alocuciones de bienvenida en el acto de apertura corrieron a cargo del Ministro de Industria y Comercio del Japón, y del Presidente del Comité de Organización, Sr. Hidehiro INOUYE, a los que respondió Mr. J. H. WALKER, de los Estados Unidos. Pronunciaron discursos de apertura Sir F. M. LEA, de Inglaterra, y el Profesor Shoichiro NAGAY, de la Uniersidad de Tokyo.

\section{ORGANIZACION Y DESARROLLO DEL SYMPOSIO}

\subsection{Trabajos presentados}

Los trabajos aportados al V-ISCC se encuadraron en dos grandes grupos, a efectos de su presentación y publicación preliminar: Trabajos Principales y Trabajos Suplemen- 
tarios ("Principal Papers" y "Suplementary Papers"). Unos y otros se incluyeron en una de las cuatro Partes (la correspondiente en cada caso), establecidas al efecto, con arreglo a los contenidos siguientes:

Parte I: Química del Clínker de Cemento.

Parte II: Hidratación de los Cementos.

Parte III: Propiedades de la Pasta de Cemento y del Hormigón.

Parte IV: Aditivos y Cementos Especiales.

Cada una de estas Partes Generales se subdividió en varias Sesiones, según los Temas. Los títulos de estos Temas y, por lo tanto, los que encabezaban cada Sesión, se hicieron coincidir con los títulos de los Trabajos Principales, que fueron en total 20 y están indicados en el esquema del Cuadro 1.

En cada Sesión se presentaron, además de los Trabajos Principales de la misma, los correspondientes Trabajos Suplementarios, que en total fueron 124, distribuidos en la forma indicada en el Cuadro 1.

\subsection{Mecánica del Symposio}

Se celebraron Sesiones durante la mañana y la tarde de los días 7 a 11, ambos inclusive (con un horario de 9,30 a 12,00 y de 14,00 a 17,30 ); en total 20 , como ya queda indicado.

Cada grupo de Sesiones (de mañana o de tarde) de cada día, estuvo presidido por un Presidente y un Co-Presidente, que presentaban sucesivamente a los autores de los Trabajos Principales; a los Informadores Generales de los Grupos de Trabajos Suplementarios y a los autores de estos trabajos; a los autores de las Discusiones Escritas de los Trabajos Principales, y a los autores de las Discusiones Orales.

Los Trabajos Principales fueron presentados por sus autores (uno por cada uno) y la presentación de los Trabajos Suplementarios, en cada Sesión, corrió a cargo de un Informador General, que hizo globalmente la del grupo de ellos que tenía asignado.

A continuación de los Trabajos Principales y Suplementarios se presentaban las Discusiones Escritas, preparadas de antemano, las cuales sólo podían referirse a Trabajos Principales y ser presentadas a razón de una por cada miembro regular del Symposio. Seguían las Discusiones Orales, sobre Trabajos Principales o Suplementarios indistintamente, presentadas por escrito con antelación, y con un máximo de tres por cada miembro regular del Symposio. Si el horario lo permitía eran admitidas también Discusiones Orales del momento, que podían calificarse de no programadas. A unas y otras Discusiones contestaban los autores, cerrando con ello el tema debatido. Había, naturalmente, la posibilidad de continuar las Discusiones en privado, y con más extensión, si a juicio de los autores y de los que las presentaban ello era necesario.

El tiempo previsto (y hay que decir que el horario se cumplió con todo rigor) fue de 20 minutos para la presentación de cada Trabajo Principal, 15, 20 ó 25 minutos para cada Informe General relativo a 5, 6 a 8, ó 9 ó más Trabajos Suplementarios, y 20 minutos para las Discusiones Escritas y Orales de cada Sesión, incluidas las correspondientes respuestas de los respectivos autores. Entre Sesiones había pausas de 15 a 20 minutos.

El idioma oficial del Symposio fue el inglés, con traducción simultánea a dicho idioma de las intervenciones hechas en japonés por las autoridades oficiales japonesas, y con 
Cuadro 1

\begin{tabular}{|c|c|c|c|c|c|c|c|c|}
\hline $\begin{array}{l}\text { Partes } \\
\mathbf{y} \\
\text { Temas }\end{array}$ & Fechas & & Sesiones y Títulos & Autores de T.P. & $\begin{array}{c}\text { Presidente } \\
\mathbf{y} \\
\text { Co-Presidente }\end{array}$ & Número de T.S. & $\begin{array}{l}\text { Informadores } \\
\text { Generales } \\
\text { de T.S. }\end{array}$ & $\begin{array}{l}\text { Otros } \\
\text { Actos }\end{array}$ \\
\hline \multirow{4}{*}{$\begin{array}{l}\text { Parte I } \\
\\
\text { Química } \\
\text { del } \\
\text { Clínker } \\
\text { de } \\
\text { Cemento }\end{array}$} & \multirow{2}{*}{$\begin{array}{c}7 \\
\text { Octubre } \\
\\
14.00 \\
16.25\end{array}$} & & $\begin{array}{l}\text { Estructura de los Mi- } \\
\text { nerales de } 1 \text { Cemento } \\
\text { Portland. }\end{array}$ & $\begin{array}{l}\text { A. GUINIER } \\
\text { M. REGOURD } \\
\text { (Francia) }\end{array}$ & \multirow{2}{*}{\begin{tabular}{|c|} 
I-1 \& 2 \\
F. M. LEA \\
(Gran Bretaña) \\
T. YAMAUCHI \\
(Japón)
\end{tabular}} & 6 & Th. HAHN & \multirow{2}{*}{$\begin{array}{c}\text { Acto } \\
\text { de } \\
\text { Apertura } \\
10.00\end{array}$} \\
\hline & & & $\begin{array}{l}\text { Equilibrio de Fases y } \\
\text { Formación de los Mi- } \\
\text { nerales del Ce me nto } \\
\text { Portland. }\end{array}$ & $\begin{array}{l}\text { R. W. NURSE } \\
\text { (Gran Bretaña) }\end{array}$ & & 9 & $\begin{array}{l}\text { Y. SUZUKAWA } \\
\text { (Japón) }\end{array}$ & \\
\hline & \multirow{2}{*}{$\begin{array}{c}8 \\
\text { Octubre } \\
\\
9.30 \\
11.50\end{array}$} & & $\begin{array}{l}\text { Análisis del Clínker de } \\
\text { Cemento Portland. }\end{array}$ & $\begin{array}{l}\text { G. YAMAGUCHI } \\
\text { S. TAGAKI } \\
\\
\text { (Japon) }\end{array}$ & \multirow{2}{*}{$\begin{array}{c}\text { I-3 \& 4 } \\
\text { R. W. NURSE } \\
\text { (Gran Bretaña) } \\
\text { G. YAMAGUCHI } \\
\text { (Japón) }\end{array}$} & 12 & $\begin{array}{l}\text { A. E. MOORE } \\
\text { (Gran Bretaña) }\end{array}$ & \\
\hline & & & $\begin{array}{l}\text { Química de los Alumi- } \\
\text { natos Cálcicos y sus } \\
\text { Compuestos Afines. }\end{array}$ & $\begin{array}{l}\text { T. D. ROBSON } \\
\text { (Gran Bretafia) }\end{array}$ & & 2 & $\begin{array}{l}\text { P. H. HALSTEAD } \\
\text { (Gran Bretaña) }\end{array}$ & \\
\hline \multirow{5}{*}{ Parte II } & \multirow{3}{*}{$\begin{array}{c}8 \\
\text { Octubre } \\
\\
\\
14.00 \\
17.20\end{array}$} & II-1. & $\begin{array}{l}\text { Estructuras Cristalinas } \\
\text { y Propiedades de los } \\
\text { Productos de Hidrata- } \\
\text { ción del Cemento (Sili- } \\
\text { catos Cálcicos Hidrata- } \\
\text { dos). }\end{array}$ & $\begin{array}{l}\text { H. F. W. TAYLOR } \\
\text { (Gran Bretaña) }\end{array}$ & \multirow{3}{*}{$\begin{array}{cc}\text { II-1, } 2 \& 3 \\
\text { H. F. W. TAYLOR } \\
\text { (Gran Bretaña) } \\
\text { R. KONDO } \\
& \text { (Japón) }\end{array}$} & 0 & & \\
\hline & & II-2. & $\begin{array}{l}\text { Estructuras Cristalinas } \\
\text { y Propiedades de los } \\
\text { Productos de Hidrata- } \\
\text { ción del Cemento (Alu- } \\
\text { minatos y Ferritos Cál- } \\
\text { cicos Hidratados). }\end{array}$ & $\begin{array}{l}\text { H. E. SCHWIETE } \\
\text { U. LUDWIG } \\
\text { (Alemania Occ.) }\end{array}$ & & $\begin{array}{ll}7 \text { (Estructuras) } \\
6 \text { (Propiedades) }\end{array}$ & $\begin{array}{c}\text { W. LOCHER } \\
\text { (Alemania Occ.) } \\
\text { M. H. ROBERTS } \\
\text { (Gran Bretaña) }\end{array}$ & \\
\hline & & II-3. & $\begin{array}{l}\text { Equilibrio de } \begin{array}{l}\text { Fases en } \\
\text { el Sistema } \\
\text { Agua. }\end{array} \\
\end{array}$ & $\begin{array}{l}\text { P. SELIGMANN - } \\
\text { N. R. GREENING } \\
\text { (Estados Unidos) } \\
\end{array}$ & & 0 & & \\
\hline & \multirow{2}{*}{$\begin{array}{c}9 \\
\text { Octubre } \\
\\
9.30 \\
12.05\end{array}$} & II-4. & $\begin{array}{l}\text { Cinética de la Hidra- } \\
\text { tación de los Cemen- } \\
\text { tos. }\end{array}$ & $\begin{array}{l}\text { R. KONDO } \\
\text { S. UEDA }\end{array}$ & \multirow{2}{*}{ 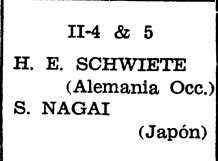 } & $\begin{array}{l}5 \text { (Mecanismo) } \\
8 \text { (Cinética) }\end{array}$ & $\begin{array}{l}\text { H. MORI (Japón) } \\
\text { H. N. STEIN } \\
\text { (Holanda) } \\
\end{array}$ & \multirow{2}{*}{$\begin{array}{c}\text { Visita } \\
a \\
\text { Industria } \\
14.00\end{array}$} \\
\hline & & II-5. & $\begin{array}{l}\text { Hidratación del Cemen- } \\
\text { to Portland. }\end{array}$ & $\begin{array}{l}\text { L. E. COPELAND } \\
\text { D. L. KANTRO } \\
\text { (Estados Unidos) }\end{array}$ & & 7 & $\begin{array}{l}\text { H. zur STRASSEN } \\
\text { (Alemania Occ.) }\end{array}$ & \\
\hline \multirow{6}{*}{ Parte III } & \multirow{2}{*}{$\begin{array}{c}10 \\
\text { Octubre } \\
\\
9.30 \\
12.15\end{array}$} & III-1. & $\begin{array}{l}\text { Estructuras y Propieda- } \\
\text { des Fisicas de la Pasta } \\
\text { de Cemento. }\end{array}$ & $\begin{array}{l}\text { G. J. VERBECK } \\
\text { R. A. HELMUTH } \\
\text { (Estados Unidos) }\end{array}$ & \multirow{2}{*}{\begin{tabular}{|l|}
\multicolumn{1}{|c|}{ III-1 \& 2} \\
G. J. VERBECK \\
(Estados Unidos) \\
M. KOKUBU \\
(Japón) \\
\end{tabular}} & $\begin{array}{l}8 \text { (Cuestiones } \\
\text { Fundamentales) } \\
7 \text { (Aplicaciones) }\end{array}$ & \begin{tabular}{|l|} 
W. C. HANSEN \\
W. L. DOLCH \\
(Estados Unidos)
\end{tabular} & \multirow{6}{*}{$\begin{array}{l} \\
\\
\\
\text { Cena } \\
19.30\end{array}$} \\
\hline & & III-2. & $\begin{array}{l}\text { Durabilidad del Hormi- } \\
\text { gón. }\end{array}$ & $\begin{array}{l}\text { O. VALENTA } \\
\text { (Checoslovaquia) }\end{array}$ & & 10 & $\begin{array}{l}\text { I. BICZOK } \\
\text { (Hungria) } \\
\end{array}$ & \\
\hline & \multirow{4}{*}{$\begin{array}{c}10 \\
\text { Octubre }\end{array}$} & III-3. & $\begin{array}{l}\text { Carbonatación del Hor- } \\
\text { migón. }\end{array}$ & $\begin{array}{l}\text { M. HAMADA } \\
\text { (Japón) } \\
\end{array}$ & \multirow{4}{*}{$\begin{array}{l}\text { R. H. MILLS } \\
\text { (Canadá) } \\
\text { T. NISHI } \\
\end{array}$} & 3 & $\begin{array}{c}\text { H. G. SMOLCZYK } \\
\text { (Alemania Occ.) }\end{array}$ & \\
\hline & & III-4-a. & $\begin{array}{l}\text { Hidratación de la Pas- } \\
\text { ta de Cemento Port- } \\
\text { land a Alta Tempera- } \\
\text { tura y a Presión At- } \\
\text { mosférica. }\end{array}$ & $\begin{array}{l}\text { G. M. IDORN } \\
\text { (Dinamarca) }\end{array}$ & & \multirow[t]{2}{*}{3} & \multirow{2}{*}{$\begin{array}{l}\text { P. J. SEREDA } \\
\text { (Canadá) }\end{array}$} & \\
\hline & & III-4-b. & $\begin{array}{l}\text { Curado del Hormigón } \\
\text { a Alta Temperatura y } \\
\text { a Presión Atmosférica. }\end{array}$ & $\begin{array}{l}\text { Yu. M. BUTT } \\
\text { v. M. KOLBASOV } \\
\text { v. v. TIMASHEV } \\
\text { (Unión Soviética) } \\
\end{array}$ & & & & \\
\hline & & III-5. & $\begin{array}{l}\text { Curado del Hormigón } \\
\text { a Alta Temperatura y } \\
\text { a Alta Presión. }\end{array}$ & $\begin{array}{l}\text { G. KALOUSEK } \\
\text { (Estados Unidos) }\end{array}$ & & 3 & $\begin{array}{l}\text { H. E. VIVIAN } \\
\text { (Australia) }\end{array}$ & \\
\hline \multirow{5}{*}{$\begin{array}{l}\text { Cementos } \\
\text { Especiales }\end{array}$} & \multirow{2}{*}{$\begin{array}{c}11 \\
\text { Octubre } \\
\\
9.30 \\
11.40\end{array}$} & IV-1. & $\begin{array}{l}\text { Empleo de Agentes } \\
\text { Tensoactivos en el Hor- } \\
\text { migón. }\end{array}$ & $\begin{array}{l}\text { R. MIELENZ } \\
\text { (Estados Unidos) }\end{array}$ & \multirow{2}{*}{$\begin{array}{c}\text { IV-1 \& } 2 \\
\text { N. STUTTERHEIM } \\
\text { (Africa del Sur) } \\
\text { S. OKUSHIMA } \\
\text { (Japón) } \\
\end{array}$} & 5 & 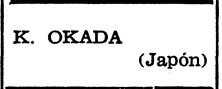 & \multirow[b]{5}{*}{$\begin{array}{l}\text { Acto } \\
\text { de } \\
\text { Clausura } \\
18.00\end{array}$} \\
\hline & & IV-2. & $\begin{array}{l}\text { Cenizas Volantes y Ce- } \\
\text { mentos con Cenizas Vo- } \\
\text { lantes }\end{array}$ & $\begin{array}{l}\text { M. KOKUBU } \\
\text { (Japón) }\end{array}$ & & 4 & A. JOISEL ${ }_{\text {(Francia) }}$ & \\
\hline & \multirow{3}{*}{$\begin{array}{c}11 \\
\text { Octubre } \\
\\
14.00 \\
17.40\end{array}$} & IV-3. & $\begin{array}{l}\text { Escorias y Cementos } \\
\text { con Escorias. }\end{array}$ & $\begin{array}{l}\text { F. SCHRODER } \\
\text { (Alemania Occ.) }\end{array}$ & \multirow{3}{*}{\begin{tabular}{|cc|}
\multicolumn{2}{|c|}{ IV-3, 4 \& 5} \\
J. H. WALKER \\
(Estados & Unidos) \\
K. CHUJO & \\
& (Japón)
\end{tabular}} & 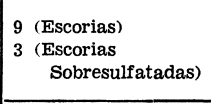 & \begin{tabular}{|c|} 
R. SERSALE \\
(Italia) \\
s. K. CHOPRA \\
(India) \\
\end{tabular} & \\
\hline & & IV-4. & Cementos Expansivos. & $\begin{array}{l}\text { P. P. BUDNIKOV } \\
\text { I. V. KRAVCHENKO } \\
\text { (Unión Soviética) } \\
\end{array}$ & & 7 & $\begin{array}{l}\text { G. J. VERBECK } \\
\text { (Estados Unidos) }\end{array}$ & \\
\hline & & IV-5. & $\begin{array}{l}\text { El Yes o Subproducto } \\
\text { de V ar i a Industrias } \\
\text { Químicas como Retar- } \\
\text { dador del Fraguado del } \\
\text { Cemento. }\end{array}$ & $\begin{array}{l}\text { K. MURAKAMI } \\
\text { (Japón) }\end{array}$ & & 0 & & \\
\hline Total & & & 20 Sesiones & 20 Trabajos & 16 Personas & 124 Trabajos & 20 Personas & \\
\hline
\end{tabular}


Cuadro 2

Horario de las Sesiones de Trabajo y otros actos.

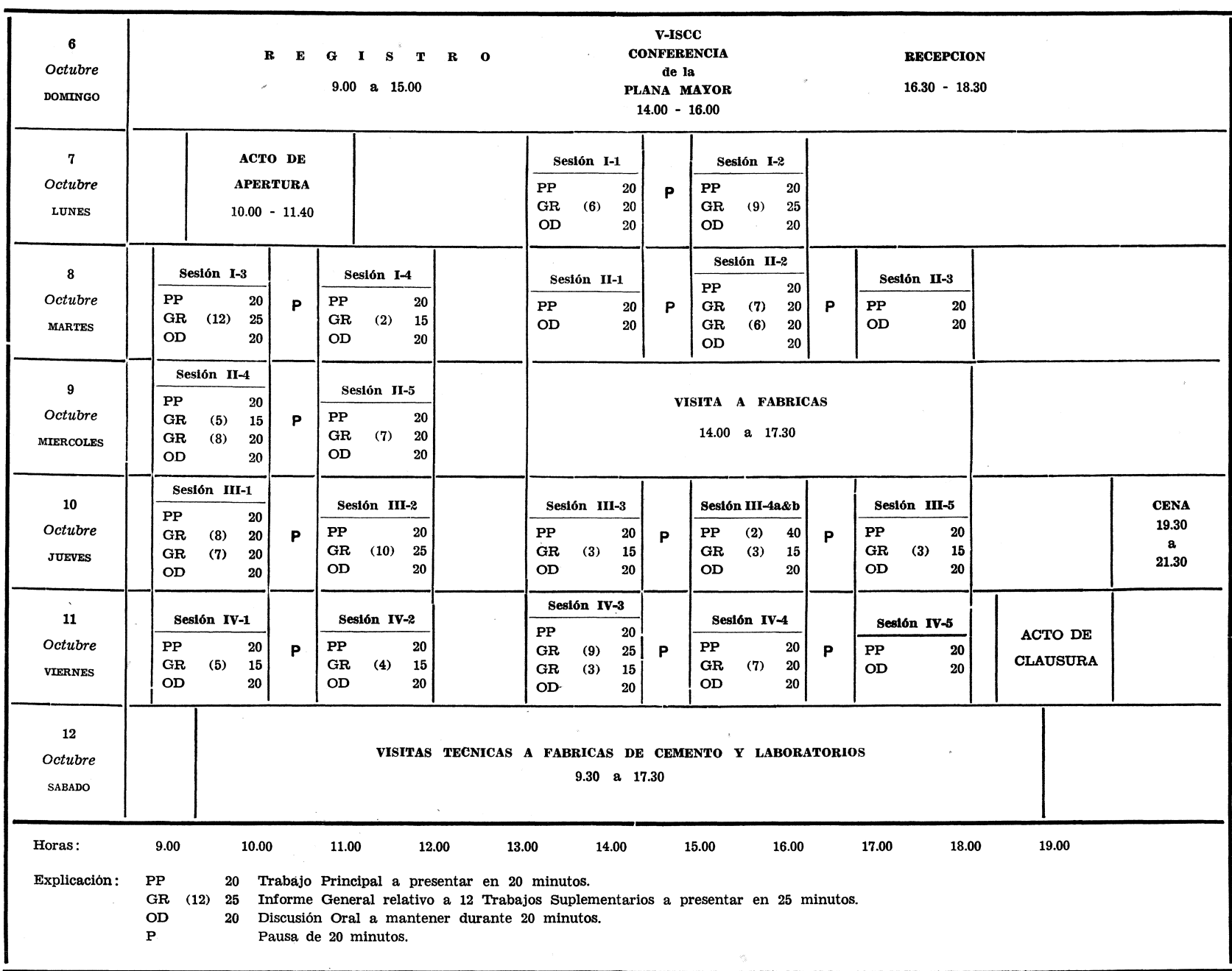


traducción simultánea al japonés de las versiones inglesas, para el mejor servicio y marcha del Symposio.

\subsection{Esquemas del Symposio}

De acuerdo con lo que antecede, los esquemas con arreglo a los cuales se desarrolló el Symposio fueron los que se indican en los Cuadros 1 y 2.

\subsection{Asistencia General y representación Española}

Asistieron al Symposio más de 250 participantes de unos 40 países, aparte Japón. Aproximadamente una mitad de los países asistentes presentó trabajos o tomó parte activa en el Symposio, si bien no todos los autores de trabajos estuvieron presentes en el mismo. La relación de Autores y Trabajos Suplementarios, por países es la dada en el Cuadro 3.

\section{Cuadro 3}

\begin{tabular}{|c|c|c|}
\hline PAISES & AUTORES & $\begin{array}{l}\text { TRABAJOS } \\
\text { SUPLEMENTARIOS }\end{array}$ \\
\hline $\begin{array}{llllllllllll}\text { 1. } & \text { Alemania } & \ldots & \ldots & \ldots & \ldots & \ldots & \ldots & \ldots & \ldots & \ldots & \ldots\end{array}$ & 41 & 20 \\
\hline 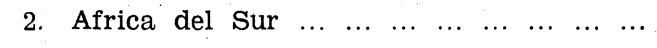 & 2 & 2 \\
\hline $\begin{array}{lllllllllllll}3 . & \text { Australia } & \ldots & \ldots & \ldots & \ldots & \ldots & \ldots & \ldots & \ldots & \ldots & \ldots\end{array}$ & 7 & 5 \\
\hline $\begin{array}{lllllllllllll}4 . & \text { Bélgica } & \ldots & \ldots & \ldots & \ldots & \ldots & \ldots & \ldots & \ldots & \ldots & \ldots & \ldots\end{array}$ & 7 & 5 \\
\hline $\begin{array}{lllllllllllll}5 . & \text { Canadá } & \ldots & \ldots & \ldots & \ldots & \ldots & \ldots & \ldots & \ldots & \ldots & \ldots & \ldots\end{array}$ & 9 & 6 \\
\hline $\begin{array}{llllllllll}\text { 6. } & \text { Checoslovaquia } & \ldots & \ldots & \ldots & \ldots & \ldots & \ldots & \ldots & \ldots\end{array}$ & 2 & 2 \\
\hline $\begin{array}{lllllllllll}\text { 7. } & \text { Dinamarca } & \ldots & \ldots & \ldots & \ldots & \ldots & \ldots & \ldots & \ldots & \ldots\end{array}$ & 6 & 2 \\
\hline $\begin{array}{lllllllllll}\text { 8. } & \text { Estados } & \text { Unidos } & \ldots & \ldots & \ldots & \ldots & \ldots & \ldots & \ldots & \ldots\end{array}$ & 28 & 15 \\
\hline $\begin{array}{llllllllllll}9 . & \text { Francia } & \ldots & \ldots & \ldots & \ldots & \ldots & \ldots & \ldots & \ldots & \ldots & \ldots\end{array}$ & 11 & 6 \\
\hline $\begin{array}{llllllllll}\text { 10. Gran Bretaña } & \ldots & \ldots & \ldots & \ldots & \ldots & \ldots & \ldots & \ldots\end{array}$ & 11 & 7 \\
\hline $\begin{array}{llllllllllll}\text { 11. } & \text { Holanda } & \ldots & \ldots & \ldots & \ldots & \ldots & \ldots & \ldots & \ldots & \ldots & \ldots\end{array}$ & 4 & 2 \\
\hline $\begin{array}{llllllllllll}\text { 12. } & \text { Hungría } & \ldots & \ldots & \ldots & \ldots & \ldots & \ldots & \ldots & \ldots & \ldots & \ldots\end{array}$ & 1 & 1 \\
\hline $\begin{array}{lllllllllllll}\text { 13. } & \text { India } & \ldots & \ldots & \ldots & \ldots & \ldots & \ldots & \ldots & \ldots & \ldots & \ldots & \ldots\end{array}$ & 7 & 4 \\
\hline $\begin{array}{lllllllllllll}\text { 14. } & \text { Israel } & \ldots & \ldots & \ldots & \ldots & \ldots & \ldots & \ldots & \ldots & \ldots & \ldots & \ldots\end{array}$ & 4 & 2 \\
\hline $\begin{array}{lllllllllllll}\text { 15. } & \text { Italia } & \ldots & \ldots & \ldots & \ldots & \ldots & \ldots & \ldots & \ldots & \ldots & \ldots & \ldots\end{array}$ & 13 & 6 \\
\hline $\begin{array}{lllllllllllll}\text { 16. Japón } & \ldots & \ldots & \ldots & \ldots & \ldots & \ldots & \ldots & \ldots & \ldots & \ldots & \ldots\end{array}$ & 80 & 37 \\
\hline 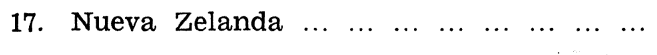 & 2 & 2 \\
\hline $\begin{array}{llllllllllll}\text { 18. } & \text { Polonia } & \ldots & \ldots & \ldots & \ldots & \ldots & \ldots & \ldots & \ldots & \ldots & \ldots\end{array}$ & 2 & 2 \\
\hline 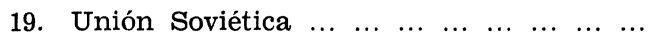 & 15 & 10 \\
\hline $\begin{array}{lllllllll}\text { TOTAL } & \ldots & \ldots & \ldots & \ldots & \ldots & \ldots & \ldots & \ldots\end{array}$ & 252 & 136 \\
\hline
\end{tabular}

Los 136 Trabajos Suplementarios anunciados en un principio quedaron reducidos definitiyamente a los 124 recogidos en el Esquema del Cuadro 1. 
Los japoneses que actuaron como autores en el Symposio fueron 7 que presentaron 5 Trabajos Principales y 65 que presentaron 37 Trabajos Suplementarios.

Los representantes españoles asistentes al Symposio con carácter de miembros regulares fueron cinco: D. Patricio PALOMAR LLOVET (acompañado de su esposa), Director Técnico de la Compañía Catalana de Cementos Portland; D. Jorge VILLAVECHIA y D. Guillermo ROSALES, de la Compañía General ASLAND; el Prof. Juan M. CORONAS, Catedrático de Química Inorgánica en la Facultad de Ciencias de la Universidad de Barcelona, y el Dr. José CALLEJA, Investigador Científico y Jefe del Departamento de Química del Instituto "Eduardo Torroja" de la Construcción y del Cemento (I.E.T.c.c.), de Madrid, autor de este trabajo.

Todos realizaron el viaje a Tokyo adscritos al grupo organizado por el CEMBUREAU, organismo internacional de cuyo Comité de Trabajo para el Estudio de los Ensayos de Cementos y Hormigones forma parte el Dr. CALLEJA. Este asistió además en concepto de miembro del I.E.T.c.c.

\subsection{Publicaciones}

Con anterioridad a la celebración del Symposio se distribuyeron entre los miembros regulares, ejemplares impresos con los textos íntegros de los Trabajos Principales, así como los "Abstracts" con los resúmenes de los Trabajos Suplementarios. Ello permitió el envío de Discusiones Escritas acerca de los Trabajos Principales. Los textos de estas Discusiones, así como los completos de los Trabajos Suplementarios fueron distribuidos en el lugar de celebración del Symposio.

El Comité de Organización publicará a la mayor brevedad las Memorias ("Proceedings") del Symposio, en las cuales figurarán los textos completos de: los Trabajos Principales y Suplementarios; las Discusiones Escritas y Orales de unos y otros; las Respuestas de los autores; los Discursos y Alocuciones de los principales oradores ; las Listas completas de países y miembros regulares participantes.

\subsection{Visitas}

Intercaladas en uno de los días de celebración del Symposio se organizaron algunas visitas a Tokyo y sus alrededores. Una de las más interesantes tuvo por objeto dar al visitante una idea de la potencialidad de la moderna industria japonesa en materia de aparatos electrónicos y ópticos. Se recorrieron, con cierto detalle, las instalaciones de las firmas SONY y CANON, situadas a la cabeza de la producción nacional japonesa en las respectivas ramas del mercado al que surten.

Aparte de éstas, se realizaron otras de carácter técnico a fábricas de cemento, a laboratorios de investigación y a institutos tecnológicos. Entre las primeras se pudieron visitar las de las firmas NIHON en Saitama y CHICHIBU en Kumagaya ; entre los segundos, el Central de la firma ONODA y el de Materiales de Construcción e Ingeniería del Instituto de Tecnología de Tokyo; y entre los terceros, el KAJIMA de Tecnología de la Construcción.

Al final del Symposio se organizaron diversas giras turísticas con visitas a distintas ciudades del archipiélago japonés. 


\section{1.\%. Participación española}

La participación española se circunscribió a la intervención del Dr. CALLEJA en la discusión del Trabajo Principal sobre "Cemento Expansivo", presentada por el Profesor P. P. BUDNIKOV y el Dr. I. V. KRAVCHENKO, de la Unión Soviética.

A propósito de las ideas originales expuestas por los autores del trabajo acerca de una teoría sobre el fenómeno de la expansión, el Dr. CALLEJA aludió a los trabajos experimentales de investigación sobre compensación de la retracción de los cementos portland mediante expansión producida y controlada por vía química. Estos trabajos se han llevado a cabo en el Departamento de Química del I.E.T.c.c. desde 1950 en adelante, habiendo dado lugar a publicaciones en los años 1956, 1962 y 1965. Como consecuencia de ellos se confirmaron plenamente hipótesis apuntadas por HEDIN en el Symposio de Química del Cemento de Estocolmo en 1938, y por LERCH en sus publicaciones en los "Proceedings" de la ASTM a partir de 1946.

A su vez, la hipótesis sustentada por el Dr. CALLEJA, llevó en el terreno práctico a la revisión y modificación de la antigua norma española para cementos, modificación que, en una u otra cuantía, ha sido introducida también y con posterioridad en otras normas extranjeras.

Se trataba en la discusión presentada, de saber si los autores del trabajo, o cualesquiera otros Delegados familiarizados con el tema, habían compulsado resultados y experiencia análogos, así como de conocer su opinión acerca de ellos y de la posibilidad de establecer un límite, a efectos prácticos de fabricación, entre cementos portland "no retractivos", "de retracción compensada" o "de expansión controlada", y cementos portland realmente expansivos, en base al contenido de yeso o anhidrita de los mismos, necesario para formar el sulfoaluminato cálcico monosulfato hidratado, en función del contenido potencial de aluminato tricálcico en un clínker dado.

En ausencia de los autores del trabajo, contestó en su nombre el Dr. O. P. PETROSSYAN, de la Unión Soviética, indicando que, en efecto, algo análogo había podido ser observado en su país, y que tal era el caso cuando se trataba de cementos portland con más de 8 por ciento de aluminato tricálcico, en los que la expansión se generaba en ellos mismos por acción de sus componentes normales, en vez de por la acción de agentes expansivos externos incorporados, como era el caso principal tratado en el trabajo de los autores. Los cementos españoles a que aludía el Dr. CALLEJA eran precisamente, de los de más alto contenido de aluminato tricálcico, sobrepasando no ya el 8 , sino incluso el 12 por ciento.

\section{IMPRESIONES DEL SYMPOSIO}

Un análisis cuantitativo de los temas tratados y de los trabajos aportados al Symposio, hecho a la vista del esquema del Cuadro 1, permite exponer las siguientes apreciaciones.

En primer lugar, la existencia de un equilibrio en cuanto a los Trabajos Principales y Suplementarios presentados en cada una de las Partes, de acuerdo con la distribución del Cuadro 4.

Esto indica que, desde Symposios anteriores, temas como el de las Adiciones y el de los Cementos Especiales, si se quiere más de aplicación y menos doctrinales que los 
clásicos de Fisicoquímica del Clínker, Hidratación del Cemento y Propiedades de la Pasta Hidratada, han llegado a alcanzar el mismo interés que éstos.

\section{CuAdro 4}

\begin{tabular}{ccccc}
\hline PARTES & $\begin{array}{c}\text { TRABAJOS } \\
\text { PRINCIPALES }\end{array}$ & \multicolumn{1}{c}{$\begin{array}{c}\text { TRABAJOS } \\
\text { SUPLEMENTARIOS }\end{array}$} & TOTAL \\
\cline { 2 - 4 } I & 4 & 29 & 33 \\
II & 5 & 33 & 38 \\
III & 6 & 34 & 40 \\
IV & 5 & 28 & 33 \\
\hline
\end{tabular}

En segundo lugar, que entre los Títulos con número global de Trabajos Suplementarios superior a 10 figuran: el Análisis del Clínker de Cemento Portland, de la Parte I, con 12; el de Estructuras Cristalinas y Propiedades de los Productos de Hidratación del Cemento (Aluminatos y Ferritos Cálcicos Hidratados), y el de la Cinética de la Hidratación de los Cementos, de la Parte II, con 13 cada uno; el de Estructura y Propiedades Físicas de la Pasta de Cemento y el de Durabilidad del Hormigón, de la Parte III, con 15 y 10, respectivamente; y el de Escorias y Cementos con Escorias, de la parte IV, con 12. De ello se deduce que entre todos destacan los dos últimos temas citados, y por razones obvias: las resistencias mecánicas de los conglomerantes hidráulicos no constituyen ya un problema para la fabricación ni para el empleo de los mismos, mientras que con carácter general el interés y la inquietud se han centrado, afortunadamente, en el más grave y difícil problema de la resistencia química de los cementos a todo plazo, en distintos medios agresivos. Esto constituye la más clara corroboración y sanción de los esfuerzos realizados por los laboratorios y centros de investigación, y por los organismos nacionales e internacionales que coordinan su labor, para profundizar en el conocimiento de las causas y mecanismos por los que se produce la destrucción química de la pasta de cemento, y de los remedios eticaces para evitarla. En línea con esta directriz está el hecho, también constatado en el Symposio de Tokyo, del incremento de interés experimentado por el tema de las Adicionea y de los Cementos Fispeciales.

De ello se deduce también la especial atención dedicada al estudio de las fases hidratadas, ferríticas y alumínicas, en contraste con la dada casi exclusivamente en otros tiempos a los silicatos hidratados, sin que ello suponga que no han sido éstos debidamente tratados en este Symposio, como lo prueba el excelente Trabajo Principal de H. F. W. TAYLOR sobre Estructuras Cristalinas y Propiedades de los Productos de Hidratación del Cemento, con especial énfasis en los Silicatos Cálcicos Hidratados.

Otra observación fácil de hacer es la referente a la atención prestada a la Cinética de los Procesos de Hidratación. En general puede decirse que el interés tanto doctrinal como práctico que tiene el conocimiento de este tema, puede verse mejor satisfecho en la actualidad gracias a las técnicas de análisis y estudio que, siendo de naturaleza física, ayudan insuperablemente a resolver el problema físico-químico de la cinética de la hidratación. Tales técnicas son la microscopía electrónica, el análisis térmico diferencial, la difracción de rayos $\mathrm{X}$, la microsonda electrónica, los métodos ultrasónicos y los de resonancia magnética nuclear o protónica. 
Algunas de estas técnicas y otras de análoga naturaleza física han contribuido también a ensanchar el campo de los trabajos de tipo analítico. Tales son la microscopía óptica, la microsonda electrónica y la termogravimetría, aplicadas al estudio de especies cristalinas del clínker, de componentes minoritarios, o de distribución de determinados componentes, como permiten apreciar algunos de los títulos de Trabajos Suplementarios presentados en la Parte I-3.

En tercer lugar, y en relación con anteriores Symposios, se ha observado en general en este de Tokyo una cierta apertura hacia temas de repercusión práctica en la utilización del cemento. Este último aspecto lo prueban, aparte de los trabajos relativos a cuestiones de durabilidad, cementos especiales y adiciones, ya glosados, los dedicados a fraguado, cambios de volumen, fisuración y tratamientos térmicos del hormigón. En cambio, en cuanto a fabricación de cemento se refiere, la Sesión I-2 incluye una parte de temas dedicados a la clinkerización de crudos, que en conjunto no llega a alcanzar el 10 por ciento del total de Trabajos Suplementarios presentados. Esto significa que los temas de Fabricación de Clínker han experimentado una regresión, en favor de los de Estructura del Clínker y de la Pasta Hidratada, y de Cinética de la Hidratación, en relación con anteriores Symposios.

\section{IMPRESIONES ACERCA DE LA INDUSTRIA DEL CEMENTO JAPONESA}

La visita efectuada a una de las tres grandes fábricas de la CHICHIBU Cement Co., Ltd., y la información general recibida con motivo de ella, permitieron adquirir una idea acerca de cuál es el estado actual y la tendencia futura previsible de la gran industria cementera japonesá. Dar cuenta detallada de ello exige más espacio del disponible aquí $y$, por otra parte, ya lo hace el autor de este informe específicamente, en otro lugar (*).

Como compendio breve de la impresión recibida puede decirse que la industria japonesa produce mucho y buen cemento, con buenos equipos y técnicas y con excelente control de procesos y calidad. $\mathrm{Y}$ también, que se trata de una industria en franco curso de expansión, con tendencia a superar producción y calidad en el futuro, a base de mejorar todos y cada uno de los aspectos técnicos de la fabricación y comerciales de cara al mercado interior y a la exportación.

La fábrica visitada es en sí un modelo de limpieza y orden, de nivel mínimo de ruidos (al menos al exterior de las naves de molienda), de escasísima polución atmosférica por polvo, y con un alto grado de automatización que permite reducir la plantilla de personal a cifras muy bajas.

Una fuerte contribución al logro de tal estado de cosas se debe, sin duda alguna, a la Asociación de Fabricantes de Cemento del Japón, que con su personal, estructura y laboratorios se halla en cuanto a madurez en investigación en todos los campos del cemento, a la altura que le corresponde por la magnitud de la industria que la respalda.

\section{IMPRESIONES ACERCA DE LA INVESTIGACION PRIVADA}

La ONODA Cement Co. Ltd., es una de las 21 empresas miembro de la Asociación de Fabricantes de Cemento del Japón. Además de producir cemento, fabrica también de.

(*) J. Calleja: La Industria del Cemento en el Japón, 1968 (I.E.T.c.c.), en este mismo número. 
rivados de éste, tales como piezas de amianto-cemento coloreadas y otros materiales y elementos de construcción, abonos y fertilizantes, y otros prcductos químicos.

Al servicio de todo este complejo tiene un laboratorio de investigación y ensayos que fue objeto de una visita, el cual ocupa la totalidad de un gran edificio de varias plantas en Tokyo. Llama la atención, no sólo la amplitud de las instalaciones y la variedad de técnicas de trabajo y estudio, sino la concepción de todo ello: pueden pasar como pertenecientes a un centro oficial de investigación en el que se cuiden al máximo los más insignificantes detalles de todo orden. Concretamente llama la atención el número y capacidad de las cámaras acondicionadas en cuanto a temperatura y humedad, entre márgenes muy amplios de una y otra variable, para la conservación, tratamiento y ensayo de diversos materiales y probetas.

Parece ser, por los datos recibidos, que tal tipo de laboratorios o centros de investigación y estudio, pertenecientes a grandes empresas o grupos de ellas, o patrocinados por las mismas, sor. frecuentes en el país.

\section{IMPRESIONES DE LAS INDUSTRIAS DEL RAMO DE LA CONSTRUCCION}

Entre Osaka y Kobe, y cerca de este último puerto, el segundo en importancia del Japón, después de Yokoama, el autor tuvo ocasión de visitar una fábrica de productos de amianto-cemento y una central preparadora de hormigón.

La primera es de superior categoría, y en el coloquio, amablemente ofrecido por sus directivos y técnicos, hubo ocasión de formular algunas preguntas acerca de ciertas dificultades que suelen ser frecuentes en este tipo de fabricación y que dan lugar a determinados defectos de las piezas fabricadas. Tales dificultades y defectos parecían ser, en general, desconocidos en la fábrica.

$\mathrm{El}$ aspecto de los productos de amianto-cemento era, por expresarlo de algún modo, algo más basto que el que normalmente se suele observar, tanto en cuanto a rugosidad superficial, como en cuanto a la apariencia del corte o sección partida de las placas. En una palabra, daban la impresión de contener una mayor proporción de amianto y, en consecuencia, una menor proporción de pasta cementante que los productos que se acostumbra a ver. Naturalmente que, si ello es así (y como es lógico no se pudo comprobar en la visita), todas las dificultades que pudieran surgir de una mayor proporción de pasta de cemento, como pueden ser movimientos retractivos y expansivos, reversibles o irreversibles, de naturaleza térmica, higrométrica o mixta, con sus consecuencias, quedarían muy atenuados, y más aún por la mayor trabazón y elasticidad que a la estructura da el mayor contenido de amianto.

En cuanto a las centrales preparadoras del hormigón, en Japón, como en tantos otros países incluido el nuestro, se han desarrollado y extendido muy rápidamente en los últimos años. La central visitada es una más, moderna y de gran capacidad, con cuidada selección y clasificación granulométrica de áridos no lavados, sin ninguna característica especial, a excepción del empleo de adítivos adecuados con distintas finalidades específicas.

Un hecho que llama la atención en las grandes ciudades (Tokyo, Kobe, Osaka, etc.) es la abundancia de grandes edificios modernos en los que el hormigón visto, formando parte de elementos estructurales y a veces meramente decorativos, constituye la nota más destacada. En muchos de estos edificios tales elementos decorativos, y en ocasiones también los estructurales, están concebidos y diseñados de tal manera que contribuyen a dar 
un cierto aire exótico a las edificaciones, en las que se conjugan en buena armonía el modernismo y la funcionalidad con las suaves líneas de la típica y ancestral construcción clásica japonesa.

\section{CONCLUSIONES}

Las que pueden sacarse de la precedente visión panorámica del Symposio, y de las visiones más parciales acerca de la investigación y de las industrias del cemento y de la construcción del Japón son las siguientes:

Japón, y concretamente Tokyo, han constituido un marco dignísimo para la celebración del Quinto Symposio Internacional de Química del Cemento. La organización, cuidada hasta el máximo en todos los detalles, ha sido decisiva para el rotundo éxito del Symposio. La contribución japonesa al mismo ha sido muy notable en calidad y cantidad, en cuanto al número de Trabajos Principales y Suplementarios presentados.

La Asociación de Fabricantes de Cemento del Japón puede, en buena ley, apuntarse el tanto de este éxito, lo cual es explicable a la vista de su estructura y de las entidades $\mathrm{v}$ personas que la integran.

El Symposio en sí ha destacado por el número de trabajos presentados, con predominio de los dedicados al estudio de la Hidratación (Cinética y Productos), de la Estructura del Clínker y de la Estructura de la Pasta Hidratada. También han hecho un cierto acto de presencia los temas analíticos y los de aplicación práctica en el campo de la utilización del cemento, tales como Durabilidad, Aditivos, Fraguado, Estabilidad y Cementos Especiales. En cambio, se ha registrado una presencia muy restringida de temas concernientes a los distintos procesos de la fabricación del cemento. La explicación puede residir en el grande y rápido desarrollo de técnicas y equipos que facilitan la investigación fructífera en los campos mencionados en primer lugar, lo cual es sin duda un aliciente para dirigir la atención de los centros de investigación y, más aún, la de los propios investigadores, hacia unos campos en los que, debido a las facilidades prestadas por dichas técnicas, el éxito es más seguro con menos esfuerzo, lo cual no priva a los temas de fabricación del gran interés de todo orden que siempre tienen.

La industria del cemento del Japón ocupa uno de los primerísimos puestos a la cabeza de la mundial, no sólo por su producción, sino también por la modernidad de sus instalaciones, por el interés primordial que presta a la investigación sobre el cemento, de lo que es prueba excepcional el propio Symposio, por las perspectivas futuras que permite vislumbrar en cuanto a automatización, productividad y mejora de las condiciones de trabajo, y por la atención especial que dedica a la calidad, al mercado, al transporte y a las nuevas vías de utilización del cemento.

A tenor de esto, se ha producido y se sigue produciendo un rápido y creciente desarrollo de las industrias de la construcción derivadas del cemento, en las que el control, el estudio y la investigación ocupan también el lugar que les corresponde. 